\title{
Recoil polarization measurements
}

\author{
Kai-Thomas Brinkmann ${ }^{1, \star}$ \\ ${ }^{1}$ II. Physikalisches Institut, Justus-Liebig-Universität Gießen
}

\begin{abstract}
Polarization observables in photon-induced meson production off nucleons have long been recognized to hold the promise of a detailed understanding of the excited states in the excitation spectrum of the nucleon. Photon beam and proton target polarization are routinely used at the ELSA facility in the Crystal Barrel/TAPS experiment and have yielded a wealth of data on contributing partial waves and nucleon resonances. A detector study on how to complement these ongoing studies by recoil polarization measurements that offer an orthogonal approach with otherwise unmeasurable observables in the field of non-strange meson photoproduction has been performed.

Building on experience with silicon detectors operated in the photon beamline environment, first possible layouts of Si detector telescopes for recoil protons were developed. Various geometries, e.g. Archimedean spiral design of annular sensors, sector shapes and rectangular sensors were studied and have been used during test measurements. A prototype for the recoil polarimeter was built and subjected to performance tests in protonproton scattering at the COSY-accelerator in Jülich.
\end{abstract}

\section{Introduction}

Polarization observables in photon-induced meson production off nucleons hold the promise of a detailed understanding of the excited states in the nucleon spectrum. Photon beam and proton target polarization are routinely used at the ELSA facility [1] in the Crystal Barrel/TAPS experiment [2] and have yielded a wealth of data on contributing partial waves and nucleon resonances. These studies could be complemented by recoil polarization studies that offer an orthogonal approach with otherwise unmeasurable observables in the field of non-strange meson photoproduction. These can provide a crucial test of as well as further constraints on the partial wave analyses of the reaction channels with the largest cross sections.

In this project, experience with silicon detectors operated in the photon beamline environment was gathered and first possible layouts of $\mathrm{Si}$ recoil detector telescopes for recoil protons have been developed. Various geometries, e.g. Archimedean spiral design of annular sensors, sector shapes and rectangular sensors were studied and used during test measurements. Possible use of the hardware in experiments such as those involving strangeness degrees of freedom can be envisaged, since the very high spatial resolution of the silicon sensors greatly improves the identification of secondary decay vertices.

The goal of this project was the development of instrumentation for the improvement of the data base on polarization observables that require measurement of the proton polarization in the final state, e.g. in $\gamma p \rightarrow p \pi^{0}$ and $\gamma p \rightarrow p \eta$. These data may have significant impact on

\footnotetext{
^e-mail: Kai-Thomas.Brinkmann@exp2.physik.uni-giessen.de
}

the PWA analyses since the recoil polarization degree of freedom in non-strange photoproduction has not yet been explored in detail.

Available data on pion and eta production to date cover total and differential cross sections over a wide range in energy with high statistical accuracy and small systematic errors as well as single polarization data with linearly polarized photons. Experiments using polarized targets and beams to measure double-polarization observables have, among others, been performed by CBELSA/TAPS [2].

As an example for the possible impact of polarization observables, Fig. 1 shows predictions for the proton recoil polarization of $\gamma p \rightarrow p \pi^{0}$ for MAID [3], SAID [4] and the Bonn-Gatchina partial wave analysis solutions for photon energies from $300 \mathrm{MeV}$ to $3.2 \mathrm{GeV}$ in 100 $\mathrm{MeV}$ steps. Large differences are visible at high energies beyond the reach of MAID, while existing data [for a recent summary, also refer to the SAID homepage at: http://gwdac.phys.gwu.edu/] at the lower energies drive the agreement of all three approaches. This effect becomes even more pronounced when polarization transfer observables that connect the spin orientation of the incoming photon with that of the outgoing proton are considered. In short, sensitivity to the solutions is large in particular for the double-polarization observables where almost no data are available. Obviously, a measurement of these observables can have a large impact on the PWA solutions and in turn on the physics in various partial waves and, thus, a large impact on our understanding of baryon resonances.

Because of the complete lack of experimental data for the $\gamma p \rightarrow p \eta$ recoil polarization (Fig. 2), huge differences between the different approaches become visible already 


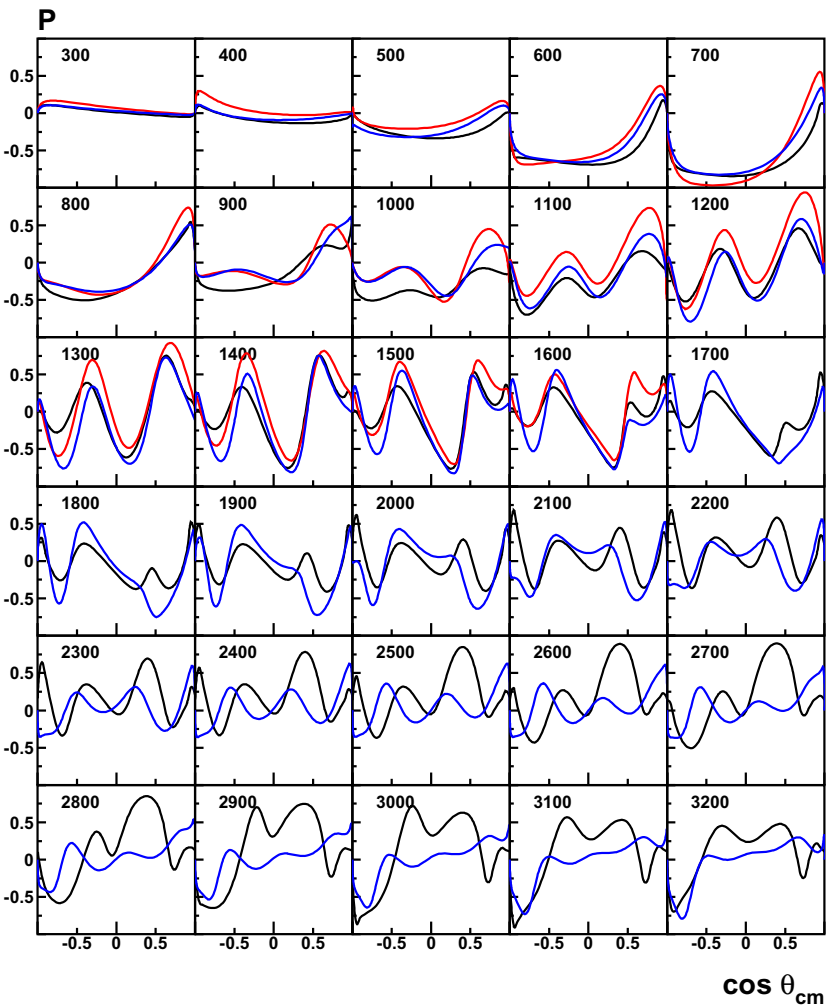

Figure 1. Angular distribution of recoil polarization $\mathrm{P}$ in $\gamma p \rightarrow p \eta$ as predicted by different models. MAID (red line), SAID (blue line) and Bonn-Gatchina PWA (black line). Differences increase with increasing beam energy, in particular where experimental data become increasingly sparse.

at small excess energies above threshold. The discrepancy is not only in the overall magnitude of the predicted polarization of the recoil proton but often completely different shapes with large absolute values and different signs occur.

The present project resulted in the realization of a detector prototype, which was used to demonstrate the feasibility of proton detection in the ELSA environment. The very high position resolution of the sensors used may prove advantageous in a variety of cases from recoil studies to hyperon identification. The detector setup that was developed can be deployed at CBElsa as well as the BGOOD set-up [5].

\section{Detector Hardware Studies}

A first set of studies was aimed at sensor development and validation for measurements of recoil protons under the special conditions of a tagged-photon beam facility. To this end, several detector layouts were studied in a variety of experimental conditions. In a first campaign of experiments the overall applicability of Si technology close the photon beam was established. Then, a first sensor, whose shape and dimensions are well suited for the geometry at hand, was brought into operation but found ill-suited for the harsh environment. Using large-area double-sided silicon strip sensors, which proved to be sufficiently radiationhard for operation in or very close to the photon beam,

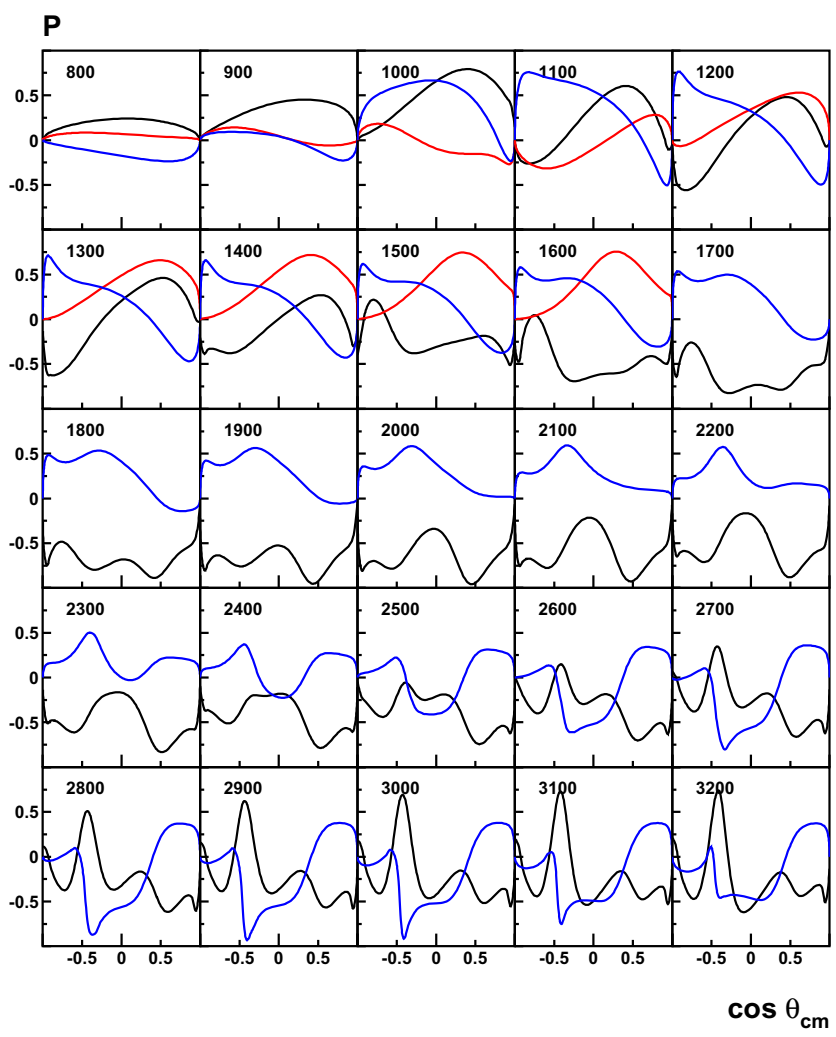

Figure 2. Angular distribution of recoil polarization $\mathrm{P}$ in $\gamma p \rightarrow p \eta$ as predicted by different models. MAID (red line), SAID (blue line) and Bonn-Gatchina PWA (black line).

an optimized layout was developed and a prototype setup built. This was subjected to extensive testing and its suitability was verified in simulations. Parallel to the detector studies, readout and data acquisition for the new addition to the experimental capabilities at ELSA was developed.

\subsection{Photon Beam Compliance}

Small silicon test sensors with an area of $2 \times 2 \mathrm{~cm}^{2}$ and a thickness of $300 \mu \mathrm{m}$ were placed in the photon beam behind the Crystal Barrel detector and their long-term properties were studied. Estimates of the photon flux and the radiation load on the detectors had been made prior to these measurements. According to these, the aging of the detectors was expected to be of no concern on typical experiment timescales, but the charged-particle background due to conversion is very difficult to estimate theoretically. The development of the detector reverse current during a long irradiation period is depicted in Fig. 3. It is dominated by the short-term changes induced by variations of the ambient temperature. Two regions with a clear correlation of photon flux (rapidly increased integral, green line) and reverse current are marked with red circles. The diagram also shows that the increase in reverse current in direct response to the irradiation is at least partly reversable through annealing. 


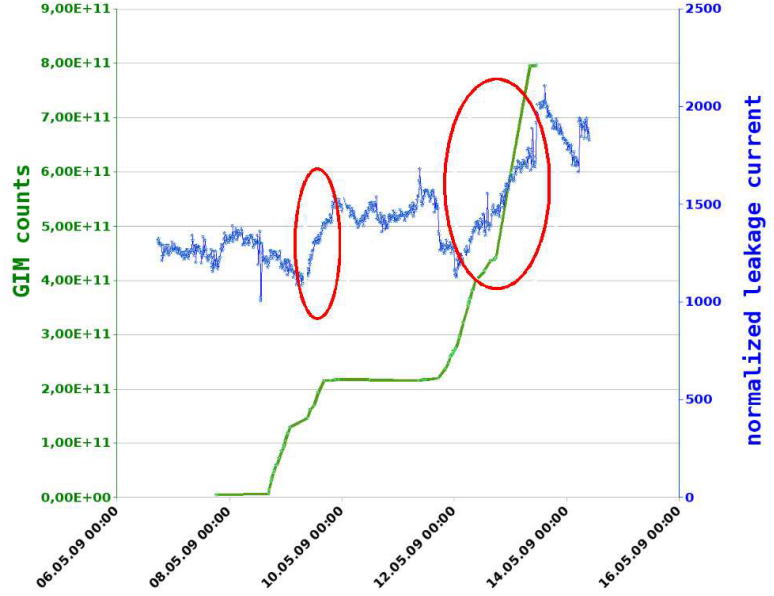

Figure 3. Development of the reverse current of a typical test detector during an irradiation period. The short-term fluctuations dominate. These are mainly due to the temperature changes. Two regions with rapid changes in reverse current (indicated by the red circles) show the correlation with the photon flux as also shown in the figure (green line, measured with the forward flux monitor GIM.)

\subsection{Test of Annular Sensors}

Having thus proven the basic applicability of Si sensors in the experiments, several detector technologies were selected for further investigation. Annular detectors with $r / \phi$ granularity are best suited to match the geometrical constraints of an experiment that aims at detection of particles close to $0^{\circ}$. Hence, an Archimedean spiral design available from a vendor (Micron Semiconductors, GB) and used in similar setups at COSY [6] was chosen as one of the possible viable options.

The sensors feature an active diameter of almost $70 \mathrm{~mm}$ and a small central hole of $8 \mathrm{~mm}$ diameter. At a nominal thickness of $300 \mu \mathrm{m}$ there are 256 Archimedean spirals with opposite senses of rotation on each side. The sensors are DC-coupled and thus have rather high noise floors. Fig. 4 shows a photograph of one of the sensors, while Fig. ?? shows a typical charge spectrum. Minimum ionizing particles cannot be discriminated from the large noise component at low amplitudes but particles are clearly visible above charges of about 3 to 5 times that of average minimum ionizing. Therefore, these sensors will allow measurements of protons with few hundreds of $\mathrm{MeV}$ of kinetic energy, albeit with non-unity efficiencies due to the pedestal cuts needed. Readout has to be done using charge-sensitive preamplifiers, which require significant electrical power and are difficult to mount in close vicinity to the detectors because of the space needed and their contribution to the material budget.

However, based on the large noise floor in a laboratory environment, it is to be expected that the sensors will exhibit rapid changes of the properties and, thus, unrecoverable time-dependent radiation damage effects during operation close to the photon beam at ELSA. After qualitative confirmation of these problems, this option was not followed up further.

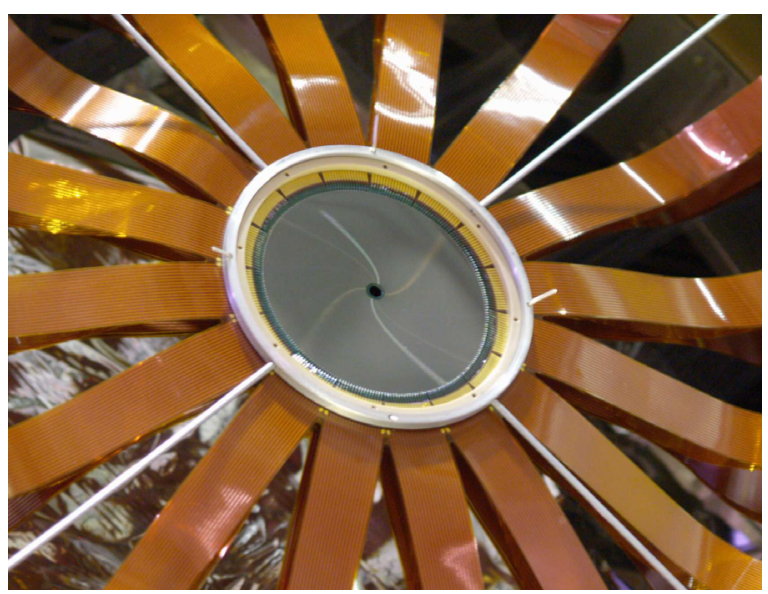

Figure 4. Photograph of one of the annular sensors.

\subsection{Setup with Square Double-Sided Sensors}

Rectangular and square double-sided AC-coupled sensors of a thickness of $300 \mu \mathrm{m}$ and a pitch (strip-to-strip distance) of $65 \mu \mathrm{m}$ offer an alternative to the DC-coupled sensors. Their layout is well advanced and measures are available to produce them with properties that make them very well suited for the task at hand. A set of these sensors was submitted to extensive electrical testing. Fig. 5 shows the capacitance measurement for a small sensor of $2 \times 2 \mathrm{~cm}^{2}$ Full depletion and, hence, optimum charge collection is reached at a voltage of $60 \mathrm{~V}$, and depletion can be achieved even after an irradiation with a total proton dose equivalent to $10 \mathrm{Mrad}$. Annealing of the sensor for increased times recovers some of the original performance with respect to small capacitance, but is not needed for proper operation. The electronic noise level of these sensors is well below 1,000 electrons compared to about 25,000 electrons in a signal for a minimum ionizing particle.

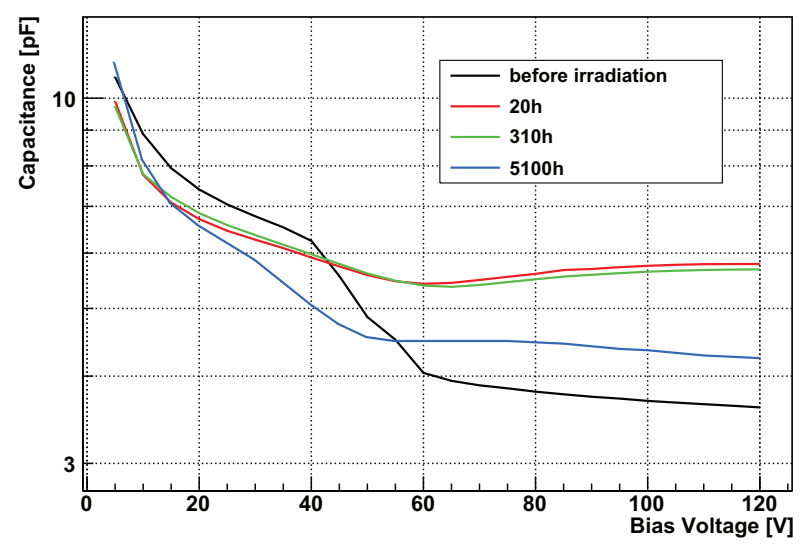

Figure 5. Bias curve for a small test sensor from the sensor run at CiS Erfurt. The sensor has been subjected to a radiation dose of $10 \mathrm{Mrad}$ after the first measurement (black curve) and then measured at different times after irradiation.

Because of the superior performance of these detectors, a setup using the square sensors was developed. It allows deployment in forward direction inside a tube small 
enough to avoid interference with the outer wire chamber tracking in the BGO-OD set-up at the cost of covering only about $60 \%$ of the area of an equivalent circular area. The conceptual layout of the holding structure and the sensors in a realistic scenario including a carbon rescatterer is shown in Fig. 6.

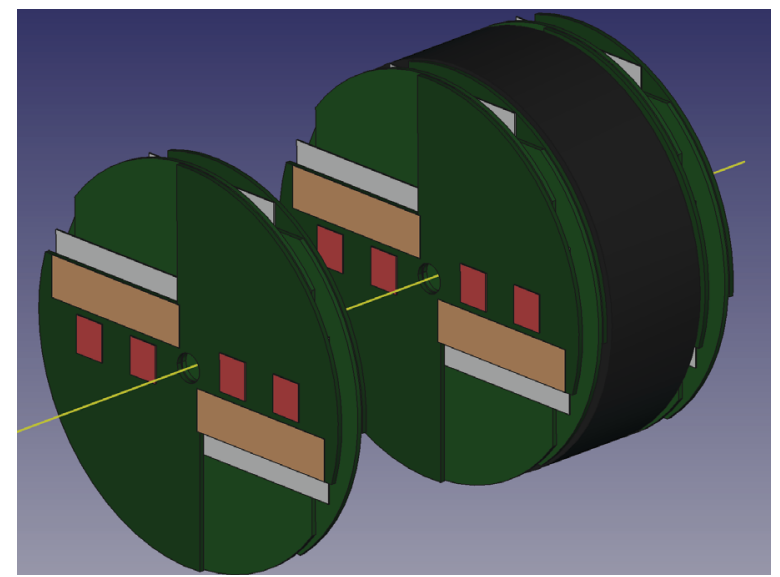

Figure 6. Layout of a mounting and support structure for double-sided square silicon sensors. All electrical services and the readout of the strips will be placed on the boards. The beam direction is indicated by the yellow line. PCBs that contain all electrical connections and serve as holding structures are shown in green, readout ASICs in dark red, and sensors are indicated by the interleaved grey areas. The black cylinder is a carbon rescatter.

This setup has been realized as shown in Fig. 7 for one sensor layer using two sensors. It is fully equipped with double-sided readout of all strips.

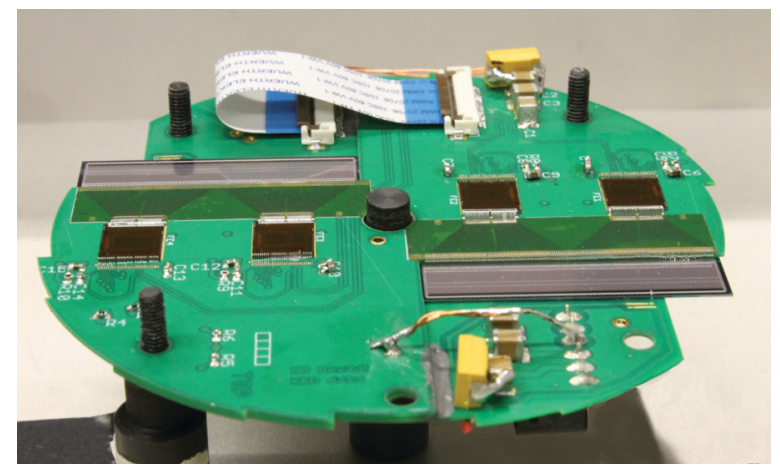

Figure 7. Photograph of one layer of the prototype recoil detector.

\subsection{Experimental Validation of the Setup}

The double-sensor design shown in the previous chapter was validated in a proton-proton scattering experiment at the COSY accelerator in Jülich [7]. The detector was placed in a symmetric position with respect to the impinging proton beam of $800 \mathrm{MeV} / \mathrm{c}$ as shown in Fig. 8. A two $\mathrm{mm}$ thick plastic sheet serves as hydrogen-rich target and the mounting plate is arranged so that the two outgoing protons from elastic scattering events will each hit one of the sensors. Two plastic scintillator detectors read out with photomultipliers serve as trigger detectors for the coincidence requirement between both protons.

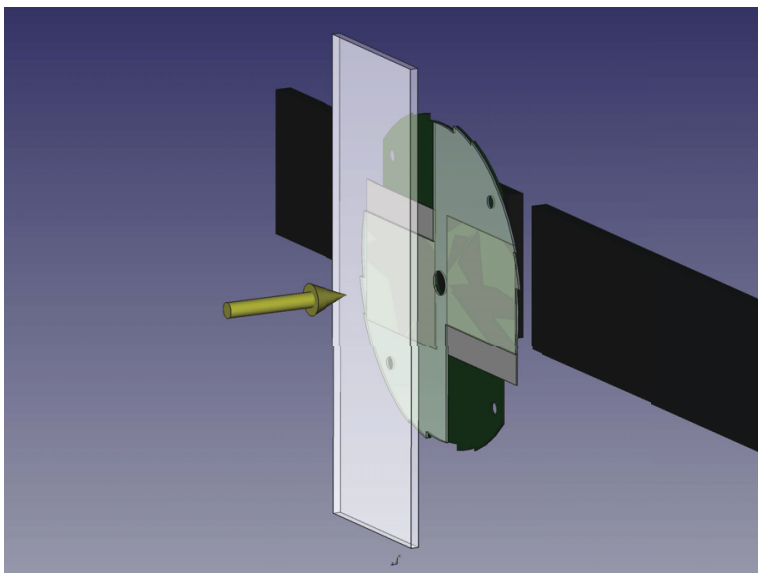

Figure 8. Layout of the COSY proton-proton scattering experiment. The protons from the accelerator hit a plastic target, behind which the sensor mount plate is placed so that the beam exits through the central hole. Two plastic scintillators (dark rectangles) serve to detect the two protons from elastic scattering events and generate a fast readout trigger.

The quality of the position measurement can be inferred from Fig. 9, where the angles of both outgoing protons detected in coincidence and derived from the position in each of the silicon detectors are shown in a 2dimensional representation. The upper frame shows data from the experiment and the lower frame is from a MonteCarlo simulation of the setup. The level of agreement is high, which demonstrates the quality of the detector setup chosen. Alongside the detector setup, all components for the readout and integration in experiments at ELSA have been developed [8] [9].

\section{Conclusions}

A detector system for the measurement of recoil polarization of protons by double-scattering was developed for use at experiments currently under way at the ELSA facility in Bonn. The sensors and their spatial arrangement were optimized for the experimental requirements of the existing large installations and the photon beam environment. All components needed for deployment including data acquisition and high-level hit reconstruction were made available. The viability of high-precision position measurements was shown in a scattering experiment at COSY.

The work reported here would not have been possible without the support from the Deutsche Forschungsgemeinschaft within the SFB/TR16.

\section{References}

[1] W. Hillert, Eur. Phys. J. A 28, 139 (2006).

[2] J. Hartmann et al., Phys. Lett. B 748, 212 (2015).

[3] D. Drechsel, S.S. Kamalov, L. Tiator, Nucl. Phys. A645, 145 (1999), [http://www.kph.unimainz.de/MAID/]. 
[4] SAID webpage, [http://gwdac.phys.gwu.edu/].

[5] B. Bantes et al., Int. J. Mod. Phys. Conf. Ser. 26, 1460093 (2014).

[6] COSY proposal 179, "Commissioning of the COSYTOF Straw Tube Tracker and the silicon-microstrip Quirl", P.Wintz, available online at http://www2.fz-

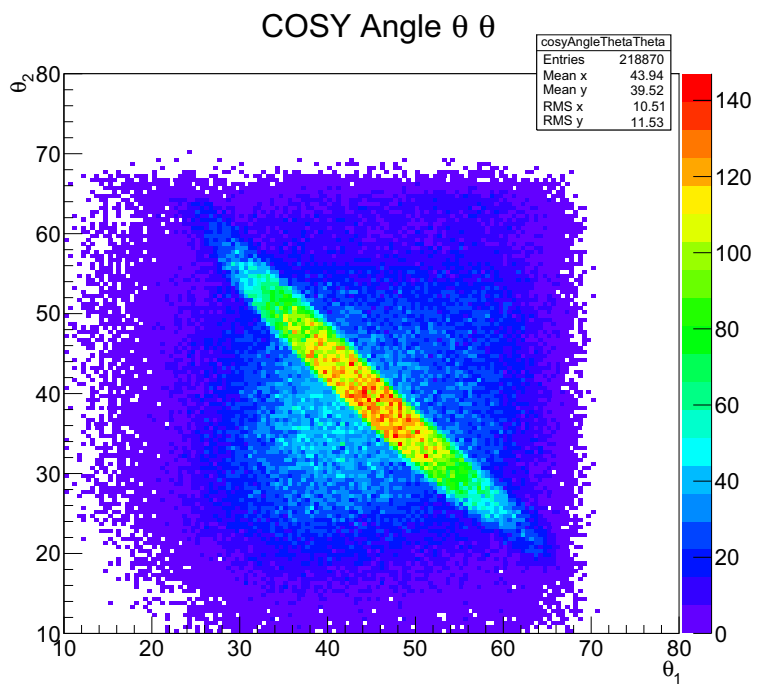

Proton Angle vs. Angle

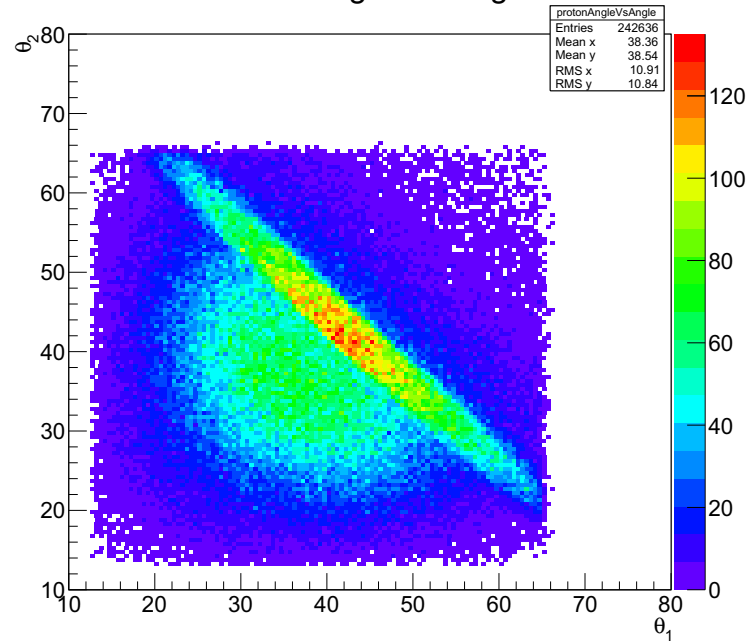

Figure 9. Data from the COSY proton-proton scattering experiment (upper frame) compared to a Monte-Carlo simulation (bottom). The angular correlation of the two outgoing protons shows a clear structure along the elastic line. Inelastic events yield smaller relative energy and thus, in general, smaller correlation angle.
juelich.de/ikp/publications/PAC34/Propo_179.pdf as of June 2012.

[7] R. Maier, Nucl. Inst. Meth. A, 390, 1 (1997).

[8] M. Becker, PhD thesis, Bonn (2014).

[9] K. Koop, PhD thesis, Bonn (2014). 